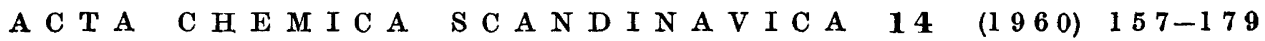

\title{
Least Squares Calculations of Equilibrium Constants for some Metal Complexes*
}

\author{
J A N R Y D B E R G **
}

Chemistry Division, Argonne National Laboratory, Lemont, Illinois, U.S.A.

\begin{abstract}
The least squares technique demonstrated in an earlier paper ${ }^{1}$ has been applied to complexes formed between a number of different organic ligands and rare earth and actinide metals. The consequences of various weighing procedures are discussed and demonstrated in the examples. A comparison is carried out between the results of the least squares calculations and the results of earlier (usually graphical) computations, and the reasons for the discrepancies are discussed.
\end{abstract}

$I_{b e}$

a recent paper ${ }^{1}$ it was demonstrated how equilibrium constants could be calculated from solvent extraction data by the methods of least squares and using high-speed digital computers. These calculations rapidly yielded the maximum number of equilibrium constants and their precision. On the other hand it was also indicated that - in the absence of very precise experimental data - the earlier graphical methods gave equilibrium constants, which cannot be supported by the results of the least squares calculations.

It was therefore felt to be of value to make a more comprehensive comparison between various methods of calculating equilibrium constants from solvent extraction data. For that purpose, a series of papers by Bernström, Dyrssen, Rydberg, et al. ${ }^{2-14}$ was selected, in which a number of different graphical computations of equilibrium constants have been used.

The principles of the various computational methods are briefly outlined below, together with the relation between the primary parameters obtained and the equilibrium constants calculated from them. The least squares method will not be described in any detail, because its use in this kind of investigations has been fully treated in earlier papers ${ }^{1,15}$. The result of this investigation is summarized in Table 1, where equilibrium constants computed by the least squares method can be compared with the constants determined by earlier methods.

* Based on work performed under the auspices of the U. S. Atomic Energy Commission.

** On leave from the University of Stockholm, and the Research Institute of National Defense, Stockholm, Sweden.

Acta Chem. Scand. 14 (1960) No. 1 


\section{OUTLINE OF VARIOUS COMPUTATIONAL PROCEDURES}

The most commonly used methods for calculation of equilibrium constants from solvent extraction data are the ligand number method (sometimes called the Bjerrum method; e.g. Refs. ${ }^{2,16,17}$ ) the limiting value method (sometimes called the Leden method; e.g. Refs. ${ }^{2,17,18}$ ) and the two-parameter method (sometimes called the Dyrssen-Sillén method ${ }^{14}$ ).

The basic equation for all the solvent extraction work discussed here is 2

$$
Q=\frac{\lambda_{\nu} \beta_{\nu}[\mathrm{A}]^{\nu}}{\sum_{0}^{N} \beta_{n}[\mathrm{~A}]^{n}}
$$

where $Q$ is the distribution ratio between the organic and aqueous phases of all mononuclear forms of the metal (charge $+\nu$ ) at constant activity factors for all species involved, [A] is the concentration of free (uncomplexed) ligand ion (here assumed to be monovalent), $\lambda_{v}$ is the partition constant of the uncharged $\mathrm{MA}_{v}$ complex between the two solvents, and $\beta_{n}$ is the gross complexity constant as defined by

$$
\beta_{n}=\frac{\left[\mathrm{MA}_{n}\right]}{[\mathrm{M}][\mathrm{A}]^{n}}=\prod_{0}^{n} k_{n}
$$

where by definition $\beta_{0}=k_{0}=1$, and $k_{n}$ is the stepwise complexity constant. For simplicity the charges of the ions have been omitted. $N$ is the maximum coordination number of $\mathbf{A}$ for $M$.

1. The ligand number method. It has been shown ${ }^{2}$ that

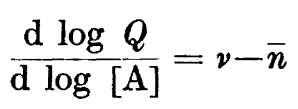

where $\bar{n}$ is the mean ligand number defined by ${ }^{16}$

$$
\bar{n}=\frac{\sum_{1}^{N} n\left[\mathrm{MA}_{n}\right]}{\sum_{0}^{N}\left[\mathrm{MA}_{n}\right]}
$$

Since $\nu$ is known, the graphical derivation of the curve $\log Q$ versus $\log [\mathrm{A}]$ will yield $\bar{n}$. Eqn. 4 can be rearranged to

$$
\sum_{0}^{N}(\bar{n}-n) \beta_{n}[\mathrm{~A}]^{n}=0 .
$$

* The symbols used here conform with those in Refs. ${ }^{1,15}$, and differ slightly from those in Refs. ${ }^{2-14}$

Acta Chem. Scand. 14 (1960) No. 1 
By choosing $N$ number of points $\bar{n}_{i}\left([\mathrm{~A}]_{i}\right)$ on the curve $\bar{n}$ versus $\log$ [A], preferably at $\bar{n}_{i}=0.5,1.5, \ldots \ldots N-0.5$, a set of $N$ equations are obtained. The solution of this set of equations by determinants using Cramer's rule is straightforward.

2. The limiting value method. Equation 1 can be rearranged to

$$
Q^{-1}=\frac{1}{\lambda_{\nu}} \sum_{N}^{0} \varphi_{\nu-n}[\mathrm{~A}]^{n-v}
$$

If, e.g. for $v=N, Q^{-1}$ is plotted against $[\mathrm{A}]^{-1}$, the limiting value when $[\mathrm{A}]^{-1} \rightarrow 0$ of the resulting curve will yield

$$
\lim _{[\mathrm{A}]^{-1} \rightarrow 0} Q^{-1}=\frac{\psi_{0}}{\lambda_{\nu}}+\frac{\psi_{1}}{\lambda_{\nu}}[\mathrm{A}]^{-1}(+\ldots)
$$

Since $\psi_{0}=1$, the intercept on the $Q^{-1}$ axes will be $1 / \lambda_{v}$, and the slope at the intercept will be $\psi_{1} / \lambda_{\nu}$. By then setting $F_{1}=\left(\frac{\lambda_{v}}{Q}-1\right)$ [A] and taking the limiting value of $F_{1}$ when $[\mathrm{A}]^{-1} \rightarrow 0$, the new intercept value will be $\psi_{1}$ and the corresponding slope $\psi_{2}$; similarly all $\psi_{n}$ and $\lambda_{\nu}$ can be determined. The relation between $\psi$ and $\beta$ is given by

$$
\psi_{\nu-n}=\beta_{n} \mid \beta_{\nu}
$$

3. The two-parameter method. It is assumed $\mathbf{1 4}$ that the relation between the equilibrium constants can be approximated to

$$
\log \beta_{N}=N p_{1}
$$

and

$$
\log k_{n} / k_{n+1}=2 p_{2}
$$

Here $10^{p_{1}}$ is called the mean complexity constant, and $10^{2 p_{2}}$ is called the mean spreading factor.

When these relations are introduced into eqn. 1, it becomes

$$
Q=\frac{h_{\nu}[\mathrm{A}]^{\nu} 10^{\nu\left[p_{1}+p_{2}(N-v)\right]}}{\sum_{0}^{N}[\mathrm{~A}]^{n} 10^{n\left[p_{1}+p_{2}(N-n)\right]}}
$$

It is seen that in addition to $\lambda_{v}$ this equation only contains two unknown parameters, $p_{1}$ and $p_{2}$. For a given combination of $\nu$ and $N$ (which are known parameters) a family of curves $\log Q / \lambda_{\nu}$ as a function of $\log \left([\mathrm{A}]+p_{1}\right)$ can be constructed, each curve representing a specific $p_{2}$ value. By placing this family of curves (e.g. suitably etched on a clear plastic ruler) over the experimental points $\log Q$ versus $\log [\mathrm{A}]$, the curve with the best fit can be found; from the curvature and intercepts on the two axes, the parameters $\lambda_{v}, p_{1}$ and $p_{2}$ are obtained. 
4. The least squares method.

Eqn. 1 can be rearranged to

where

$$
z=\sum_{0}^{N} a_{n}[\mathrm{~A}]^{n}
$$

and

$$
z=[\mathrm{A}]^{\nu} / Q
$$

$$
a_{n}=\beta_{n} / \lambda_{\nu} \beta_{v}
$$

Eqn. 11 can be solved by the method of weighted least squares using high speed digital computers 1,15 to cut down on the computation time. It will then yield $a_{n} \pm \sigma_{a_{n}}$, where $\sigma_{a_{n}}$ is the standard deviation in $a_{n}$. The weight, which has to be assigned to the $z_{i}$ values, depends on the precision of the measurements of $Q_{i}$.

Several kinds of standard errors in $Q$ have been tested here for solvent extraction data: (1) errors in $Q$ (or in $I_{\text {org }}$ and $I_{\text {aq }}$, which represent measured standardized radioactivities of the organic and aqueous phases) given in the original paper, (2) a counting error in $Q$, called $\sigma_{Q}(I)$, based on the assumption that the number of measured counts is 5 times that given for the intensities ( 5 min. measurement if $I$ is given in counts per min., cpm) and that the background error is \pm 1 (thus, e.g. $\sigma\left(I_{\mathrm{aq}}\right) \approx 1+\sqrt{I_{\mathrm{aq}} / 5}$ ), (3) a constant percentage (P) error, called $\sigma_{Q}(\%)$, making $\sigma_{Q}(\%)=\mathbf{P} Q,(4)$ errors in $Q$ caused by errors in the determination of the free ligand concentration. These kinds of errors are more extensively discussed elsewhere ${ }^{1}$.

Since the weight of a point $z_{i}\left(x_{i}\right)$ is defined here as one over the variance $\sigma_{i}{ }^{2}$ of the $z_{i}$ value, i.e. $\omega_{i}=1 / \sigma_{i}{ }^{2}$, the weight of all points can be changed by multiplying the variance with a constant factor. This will not change the $a_{n} \pm \sigma_{a_{n}}$ values, $c f$. Ref. ${ }^{15}$, but will alter the $\chi^{2}$ values $\left(e . g\right.$. Ref..$\left.^{20}\right)$, which also are calculated in the computer program ${ }^{1,15}$. Since the ratio $\chi^{2} / k$ should be within the range $0.5-1.5$ for a reasonable consistency between the experimental data and the equations used for computing $a_{n}, \chi^{2} / k$ can be brought into this range by changing the weights of the data. This procedure is used all through this investigation to see what size of standard error should have been assigned to the original data. The $k$ value is the number of degrees of freedom in the computations and is equal to $L-N-1$ for eqn. $11 ; L$ is the number of experimental points.

It is obvious that when $\sigma_{a_{n}}>a_{n}$, the $a_{n}$ value is of little statistical significance. If such an $a_{n}$ value is obtained, it is omitted from eqn. 11 and the least squares computations are repeated without it. Though this means mathematically that the particular $a_{n}$ value is put equal to zero, it should be interpreted only as a way of remowing parameters from the calculations which can only be determined very inaccurately; this usually causes only small changes in the remainning parameters (see paragraphs a and $d$ belew). Similarly, negative $a_{n}$ values are omitted, because in the deductions of the basic solvent extraction equations only positive concentrations of the complexes are assumed to exist. 


\begin{tabular}{|c|c|c|c|c|c|c|}
\hline 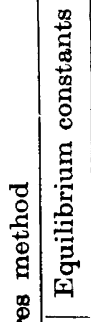 & 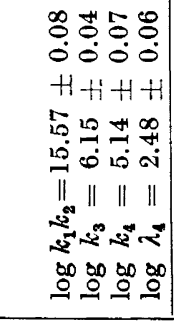 & 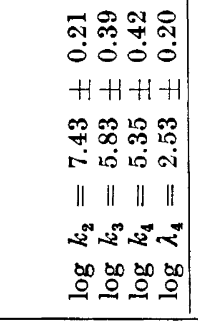 & 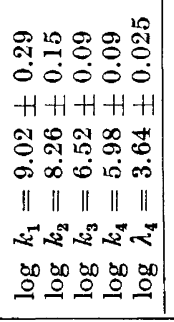 & '. & 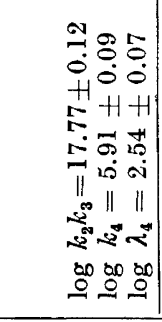 & \\
\hline 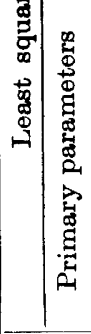 & 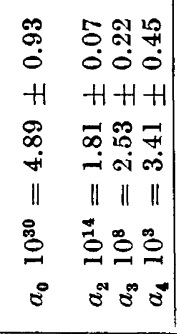 & 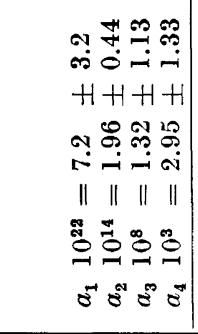 & 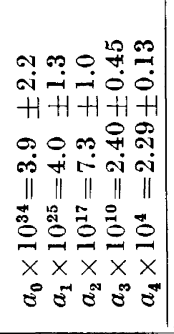 & & 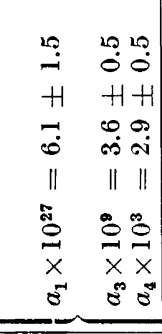 & \\
\hline 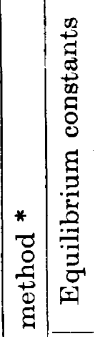 & 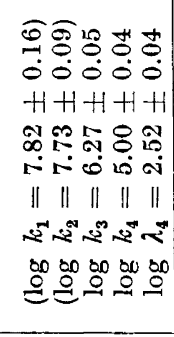 & 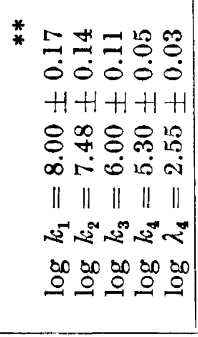 & 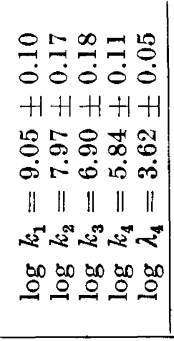 & 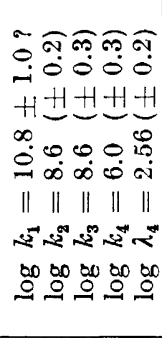 & 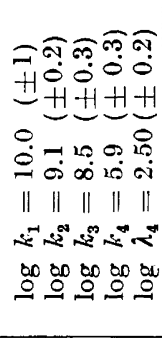 & 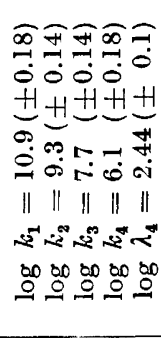 \\
\hline 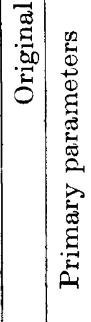 & 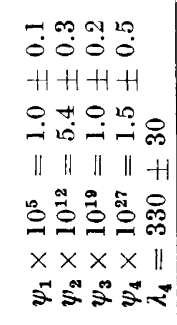 & 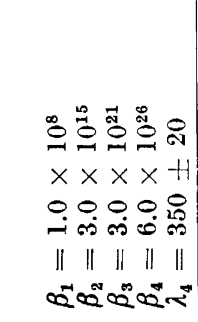 & 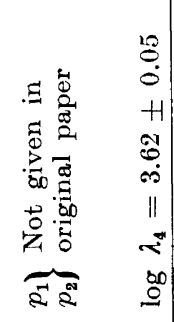 & 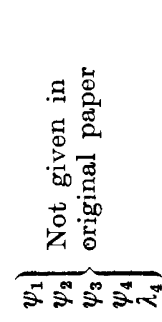 & 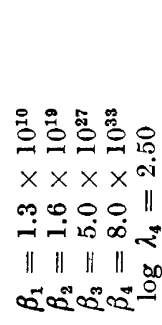 & 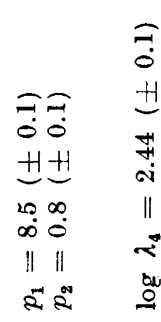 \\
\hline 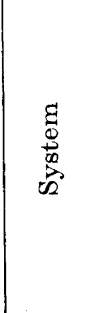 & 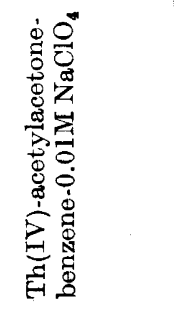 & 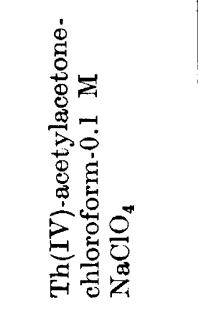 & 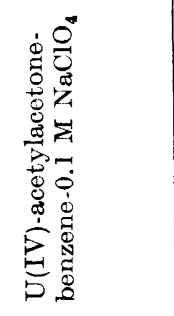 & 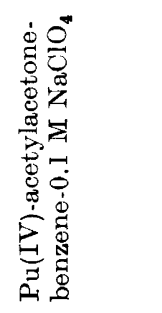 & & \\
\hline $\cos$ & $\theta$ & $\therefore$ & 0 & $\tau$ & & \\
\hline
\end{tabular}

Acta Chem. Scand. 14 (1960) No. 1 


\begin{tabular}{|c|c|c|c|c|c|}
\hline 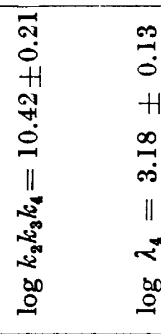 & 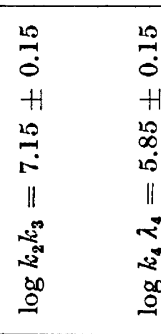 & 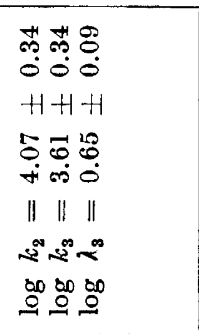 & 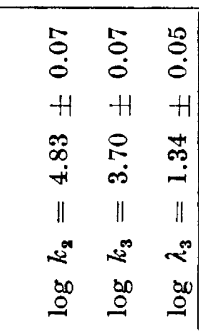 & 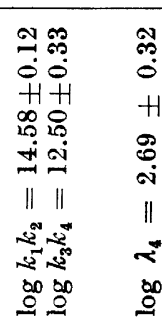 & 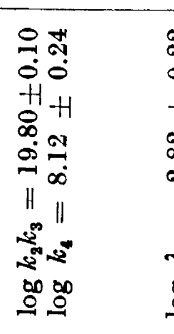 \\
\hline 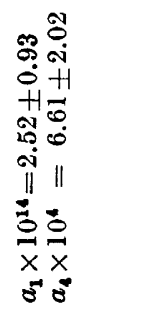 & 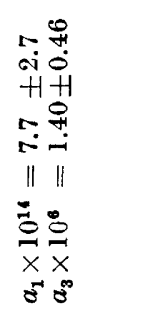 & 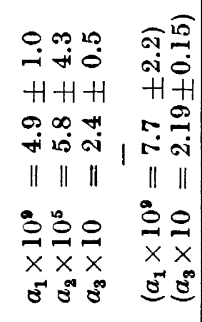 & 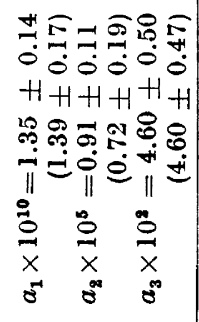 & 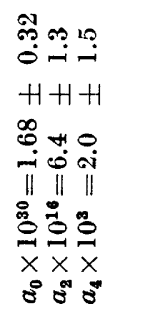 & 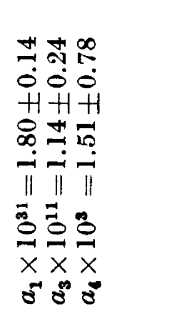 \\
\hline 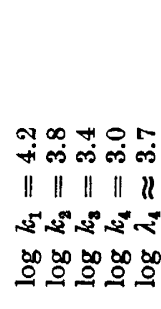 & 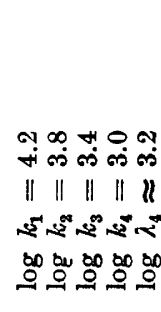 & 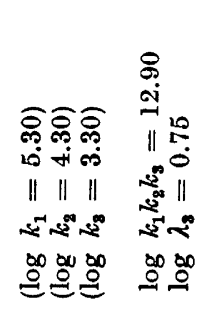 & 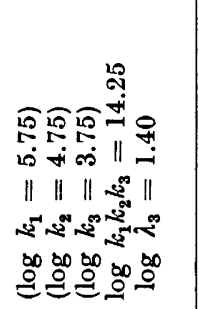 & 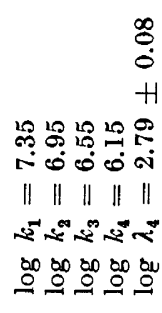 & 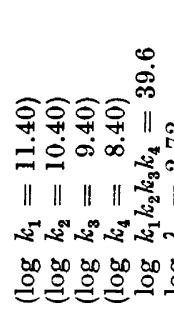 \\
\hline 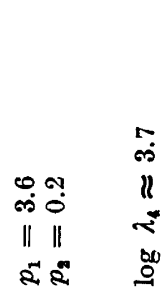 & 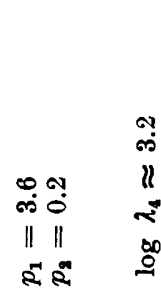 & 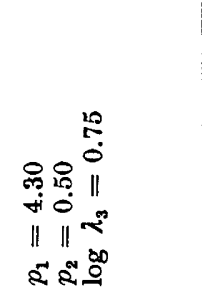 & 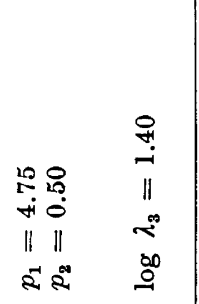 & 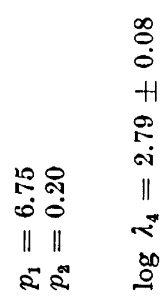 & $\begin{array}{l}0 \\
0 \\
H \\
H \\
80 \\
80 \\
00 \\
11 \\
21 \\
=2\end{array}$ \\
\hline 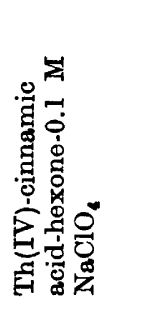 & 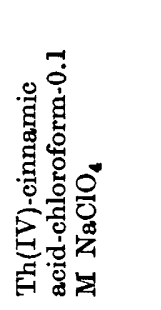 & 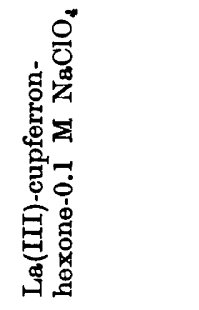 & 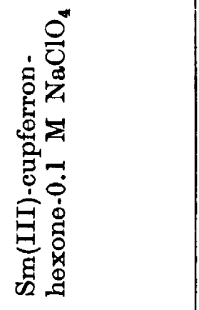 & 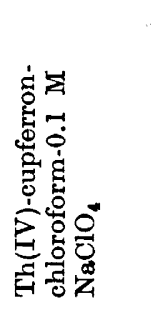 & 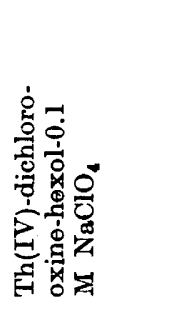 \\
\hline 0 & $4+1$ & $\infty$ & 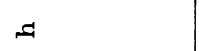 & $\rightarrow$ & $\rightarrow$ \\
\hline
\end{tabular}


E Q UIIIBRIUM C ONSTANTS

163

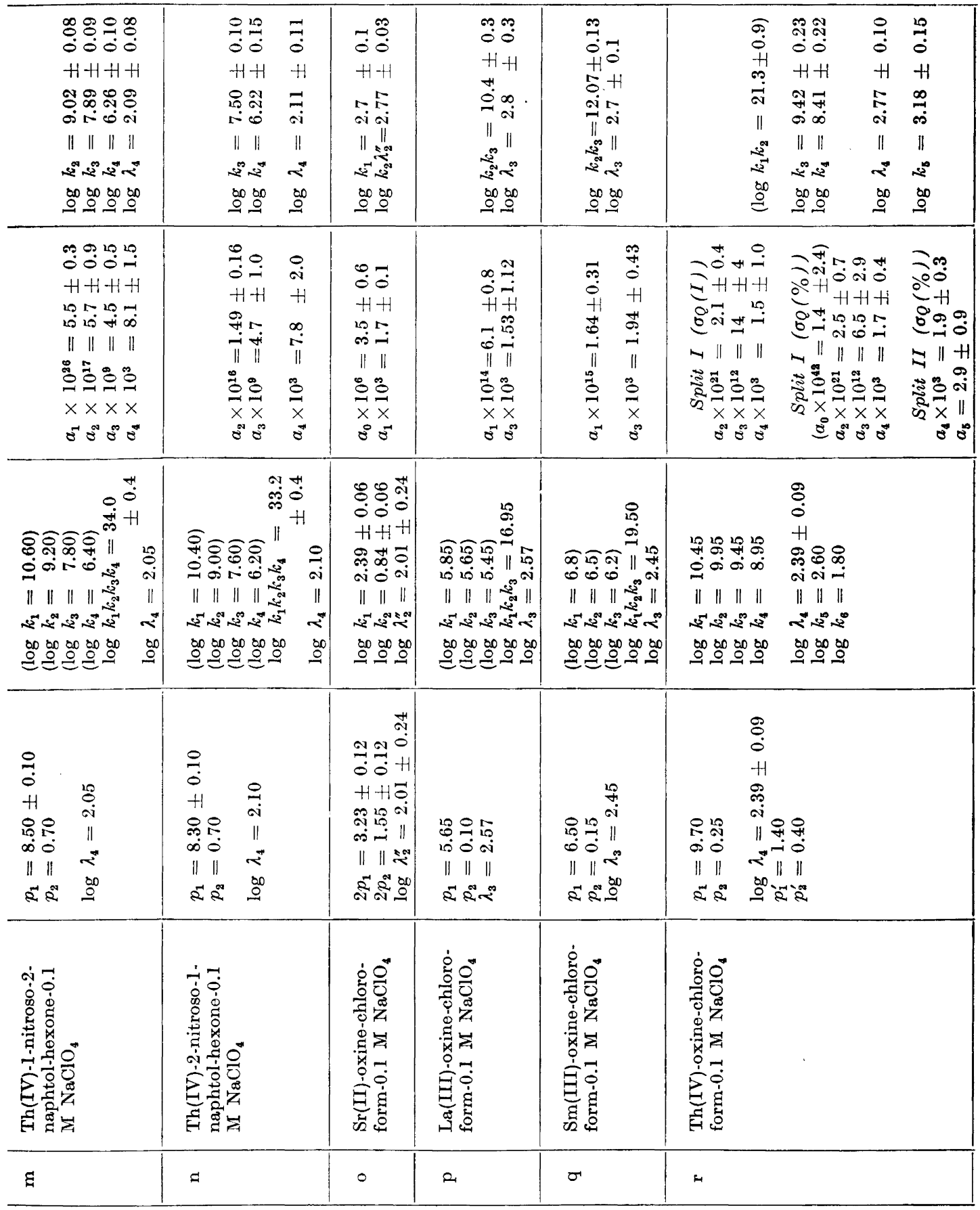

Acta Chem. Scand. 14 (1960) No. 1 


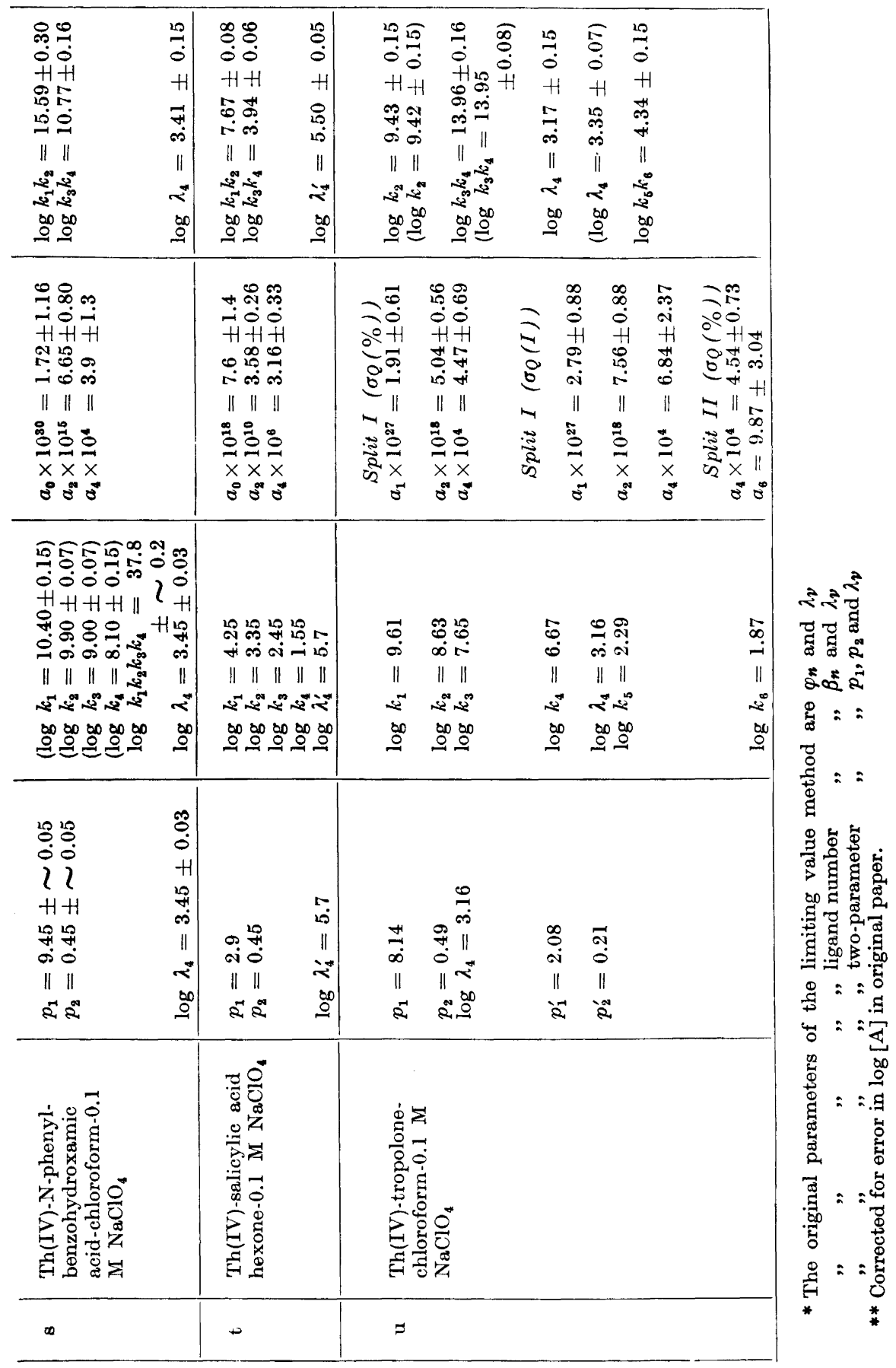

Acta Chem. Scand. 14 (1960) No. 1 


\section{RESULTS}

The solvent extraction systems investigated here are given in alphabetical order of the ligand in Table 1, which contains the computed primary parameters and the equilibrium constants obtained from them. Sometimes only the primary parameters are given in the original papers and usually without any indication of the sizes of the standard deviations. In order to facilitate a comparison between the least squares calculations and the results of the other methods in these cases, equilibrium constants have been computed from the parameters and standard deviations estimated when possible; those values which are not found in the original paper are given within parenthesis in the table.

In all systems, the least squares parameters $a_{n}$ have been used to calculate $\log Q$ as a function of $\log [\mathrm{A}]$ according to

$$
Q=[\mathrm{A}]^{v} / \sum_{0}^{N} a_{n}[\mathrm{~A}]^{n} .
$$

If nothing particular is said in the text accompanying the Tables, it is understood that the curve thus obtained goes smoothly through the experimental points with about equally many points on both sides of the curve (see also Discussion).

a. The Th $(\mathrm{IV})$-acetylacetone-benzene-0.01 $\mathrm{M} \mathrm{NaClO}_{4}$ system ${ }^{2}$. The least squares computations on this system have been discussed before ${ }^{1}$. Regardless of the kinds of errors tested for the primary data, the parameter $a_{1}$ came out negative. Omitting $a_{1}$ and using a certain combination of counting and $\mathrm{pH}$ error (see Ref. ${ }^{1}$ ), the $a_{n}$ values in Table la were obtained. (The corresponding $a_{n}$ values with all parameters are: $a_{0} 10^{30}=6.4 \pm 1.7, a_{1} 10^{22}=-1.3 \pm 1.3$, $a_{2} 10^{14}=1.94 \pm 0.15, a_{3} 10^{8}=2.34 \pm 0.29$ and $a_{4} 10^{3}=3.53 \pm 0.49$; omitting $a_{1}$, the changes in the remaining parameters are rather small, see Table la).

Though the requilibrium constants of the two computational procedures are in good agreement, the spread of the experimental data (an average standard deviation of about $25 \%$ ) prevents a separation of $k_{1}$ from $k_{2}$. With the limiting value method more constants have been calculated than are justifiable. The precision of the equilibrium constants of the limiting value method is based on graphical estimates and are obviously in good agreement with the corresponding least squares data.

b. The Th(IV)-acetylacetone-chloroform-0.1 $\mathrm{M} \mathrm{NaClO}_{4}$ system. ${ }^{3}$ In the original paper, the standard deviations of the primary data $I_{\text {org }}$ and $I_{\text {aq }}$ are given. Using these deviations, the least squares procedure yields a negative value of $a_{0}$ and with a standard deviation of $>100 \%$. Omitting this parameter, a $\chi^{2} / k$ value of 39 is obtained, indicating that the weights assigned from the errors in the primary data are incorrect (cf. Ref. ${ }^{1}$ ).

Following the procedure discussed in Ref. ${ }^{1}$ for the Th-acetylacetonebenzene case, both a counting error $\left(\sigma_{Q}(\mathrm{I})\right)$ and a ligand error $\left(\sigma_{Q}(\mathrm{~A})\right)$ was tried, separately as well as combined. With a weighing factor of 6 times the original counting error, and a ligand error corresponding to \pm 0.01 log units, 


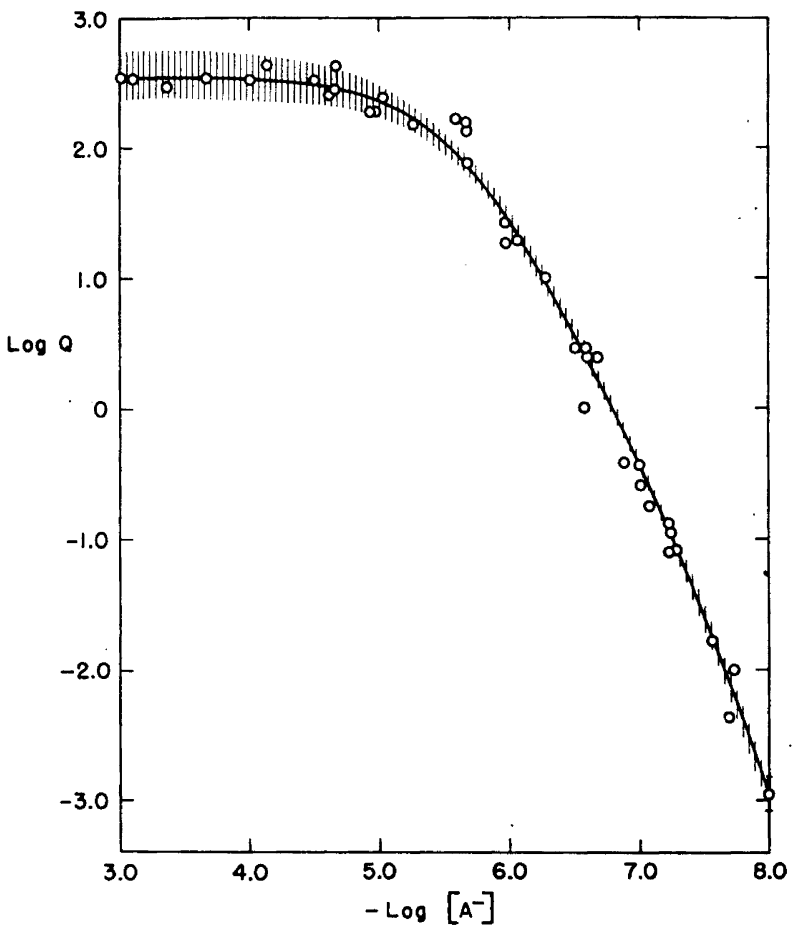

Fig. 1. The Th(IV)-acetylacetone-chloroform-0.1 $\mathrm{M} \mathrm{NaClO}$ system. The curve is computed from the least squares parameters in Table $1 \mathrm{~b}$. The shaded area indicates the precision of the curve.

$\chi^{2} / k=1.5$ was obtained; the $a_{*}$ values for this combination are given in Table $1 \mathrm{~b}$. The fact that so much larger errors had to be assigned to the data indicates that the solvent extraction procedure may involve large errors, which were not originally accounted for.

Table lb shows that though the numerical values of the equilibrium constants obtained with the two computational methods agree fairly well, the least squares method indicates that the experimental data do not allow a calculation of $k_{1}$. This is also clear from Fig. 1, where the curve computed without $k_{1}$ obviously fit the data very well. The broad band around the curve in Fig. 1 gives the precision of the curve as obtained with the least squares method. From Table $\mathrm{Ib}$ it is also seen that the estimated errors given for the equilibrium constants of the ligand number method are far too small.

c. The $U(I V)$-acetylacetone-benzene-0.1 $\mathrm{M} \mathrm{NaClO}_{4}$ system ${ }^{4,19}$. The least squares treatment of this system has recently been described ${ }^{19}$. The results are summarized in Table lc, where comparison is made only with the results of the two-parameter method, though the ligand number method was also used in the original paper ${ }^{4}$. 
Table 2. Comparison between $a_{n}$ parameter values obtained by the least squares calculations and those computed from graphically obtained equilibrium constants for the $\mathrm{Pu}(\mathrm{IV})$ acetylacetone system.

\begin{tabular}{|c|c|c|c|c|c|c|}
\hline \multirow{2}{*}{ Method } & \multicolumn{6}{|c|}{ Parameter values } \\
\hline & $\chi^{2} / k$ & $a_{0} \times 10^{37}$ & $a_{1} \times 10^{26}$ & $a_{2} \times 10^{17}$ & $a_{8} \times 10^{9}$ & $a_{4} \times 10^{3}$ \\
\hline $\begin{array}{l}\text { limiting value } \\
\text { ligand number } \\
\text { two-parameter } \\
\text { least squares } \\
\text { (5 parameters) } \\
\text { least squares } \\
\text { (3 parameters) }\end{array}$ & $\begin{array}{l}- \\
- \\
0.91 \\
1.07\end{array}$ & $\begin{array}{c}2.8 \\
1.0 \\
3.6 \\
-17 \pm 10 \\
- \\
\end{array}$ & $\begin{array}{c}1.7 \\
1.0 \\
2.9 \\
1.9 \pm 0.8 \\
0.61 \pm 0.15\end{array}$ & $\begin{array}{c}6.9 \\
1.3 \\
5.8 \\
-0.046 \pm 0.53 \\
-\end{array}$ & \begin{tabular}{|c|}
2.8 \\
4.0 \\
2.9 \\
$3.3 \pm 0.6$ \\
$3.6 \pm 0.5$ \\
\end{tabular} & $\begin{array}{c}2.8 \\
3.2 \\
3.6 \\
2.9 \pm 0.4 \\
2.9 \pm 0.5\end{array}$ \\
\hline \multicolumn{2}{|c|}{$\begin{array}{l}\text { Parameters refer to the following } \\
\text { equilibria (no index means aqueous } \\
\text { phase): }\end{array}$} & $\frac{[\mathrm{M}][\mathrm{A}]^{4}}{\left[\mathrm{MA}_{4}\right] \text { org }}$ & $\frac{[\mathrm{MA}][\mathrm{A}]^{3}}{\left[\mathrm{MA}_{4}\right] \mathrm{org}}$ & $\frac{\left[\mathrm{MA}_{2}\right][\mathrm{A}]^{2}}{\left[\overline{\mathrm{M}} \mathrm{A}_{4}\right] \text { org }}$ & $\frac{\left[\mathrm{MA}_{3}\right][\mathrm{A}]}{\left[\mathrm{MA}_{4}\right] \text { org }}$ & $\frac{\left[\mathrm{MA}_{4}\right]}{\left[\mathrm{MA}_{4}\right]_{\text {org }}}$ \\
\hline
\end{tabular}

This system is the only one in the present investigation where significant values have been obtained for all possible parameters using the least squares method. The agreement between the results of the two computational methods is fair. The precision of the equilibrium constants of the two parameter method is based on graphical estimates ${ }^{19}$.

d. The Pu(IV)-acetylacetone-benzene-0.1 $\mathrm{M} \mathrm{NaClO}_{4}$ system $^{5}$. From the spread of the experimental points, the standard deviations in $Q$ were assumed to be $20 \%$. With the least squares method this produced a $\chi^{2} / k$ value of 0.91 and the parameter values in Table 2. Omitting the negative parameters, a $\chi^{2} / k$ of 1.07 was obtained, indicating that the $20 \%$ error is a reasonable estimate.

Table Id contains the primary parameters and the equilibrium constants computed from them, including those of all three different methods given in the original paper. The precision values given within parenthesis are, for the limiting value and ligand number methods, estimates from the differences in $\beta_{n}$-values obtained with these two methods. In the two-parameter method, the precision in the primary parameters are here assumed to be \pm 0.1 .

It is seen from Table 2 that $a_{0}$ is negative with $\sigma_{a_{0}}<\left|a_{0}\right|$, which should mean that negative concentrations of either $\mathrm{Pu}^{4+}$ or $\mathrm{PuAa}_{4}$ exist. Since this cannot be true, $a_{0}$ had to be omitted with the consequences that no $k_{1}$ value can be calculated. The difficulty encountered in calculating $k_{1}$ with the limiting value method also supports that the calculation of such a value (as has been done with the ligand number and two-parameter methods) is dubious. Thus in contradiction to the graphical methods, the least squares result clearly indicates that the experimental data do not permit a calculation of $k_{1}$, the reason probably being that the concentration of $\mathrm{Pu}^{4+}$ in the experiments is too low to have any serious influence on the measurements. (It is obvious that the omission of $a_{0}$ does not mean that $\mathrm{Pu}^{4+}$ does not exist! cf. p. 160).

Acta Chem. Scand. 14 (1960) No. 1 
Since $a_{2}$ was negative and was associated with a very large standard error jt was also omitted. Of the $a_{2}$ values computed from the graphically obtained equilibrium constants (se日 Table 2), it is seen that the ligand number value is much smaller than those of the other two methods. In the original paper ${ }^{5}$, a plot of $\bar{n}$ against $\log$ [A] for $\mathrm{Pu}(\mathrm{IV})$ showed a steeper slope at $\bar{n}=2$ than was the case for the similar $\mathrm{Th}(\mathrm{IV})$ and $\mathrm{U}(\mathrm{IV})$ systems ${ }^{2-4}$, thus indicating an unexpectedly low concentration of the $\mathrm{PuA}_{2}^{2+}$ complex. The numerically low and highly uncertain value of $a_{2}$ obtained by the least squares calculation agrees well with these more visual findings on the $\bar{n}$ curve. It must therefore be concluded that in the $\mathrm{Pu}(\mathrm{IV})$ acetylacetone system the formation of the second complex, PuA $\mathbf{2}_{2}^{+}$, seems to be hindered. A "regular" behaviour of the system would give an $a_{2}$ value of about $6 \times 10^{-17}$ (the two-parameter value), while its real value probably is $<0.5 \times 10^{-17}$. A use of the $a_{2}$ value obtained by the two-parameter or limiting value methods is therefore probably very misleading.

On the other hand, it should be mentioned that both $\mathrm{Th}(\mathrm{IV})$ and $\mathrm{U}(\mathrm{IV})$ show an unusually high stability for their second acetylacetonate complex, as judged by the regular principles of the two-parameter method. The only explanation that would remove the conclusions above about the $\mathrm{PuA}_{2}^{2+}$ complex is that systematic errors are involved in the $\mathrm{Pu}(\mathrm{IV})$ system which do not appear in the Th(IV) and $\mathrm{U}(\mathrm{IV})$ systems, or vice versa. Investigations by other methods than solvent extraction may settle this question; such experiments are being planned.

e. The Th(IV)-cinnamicacid-hexone-0.1 $\mathrm{M} \mathrm{NaClO}$-system $^{6}$ As pointed out in the original paper, the data are badly scattered which makes accurate calculations of the equilibrium constants difficult. By choosing $\sigma_{Q}(\%)=$ $0.7 Q, \chi^{2} / k=0.99$ was obtained with two negative parameters $\left(a_{0}\right.$ and $\left.a_{3}\right)$ and two parameters having standard deviations $>100 \%\left(a_{2}\right.$ and $\left.a_{3}\right)$. Omitting these three parameters, $\chi^{2} / k=1.03$ is obtained with the $a_{n}$ values in Table le.

Thus the least squares method only yields two parameters, while the twoparameter method yields three parameters, that is one parameter more than the experimental data permit. Though the $k_{1} k_{2} k_{3}$ values of the two computations agree, the $\lambda_{4}$ values show a rather large discrepancy.

$f$. The Th(IV)-cinnamic acid-chloroform-0.1 $\mathrm{M} \mathrm{NaClO}_{4}$ system. ${ }^{6}$ In the least squares treatment, the parameters $a_{0}, a_{2}$, and $a_{4}$ came out negative. With these parameters omitted and $\sigma_{Q}(\%)=0.7 Q$, a $\chi^{2} / k=0.85$ was obtained; the corresponding $a_{n}$ values are given in Table lf.

In the original paper ${ }^{6}$, three parameters are obtained: $p_{1}, p_{2}$, and $\lambda_{4}$. Fig. 2 shows the curve in the original paper given with these three parameters and also the curve computed with the $a_{1}$ and $a_{3}$ parameters. It is obvious that the equilibrium constants calculated from the least squares parameters give all the information which can be obtained from the experimental data.

g. The La(III)-cupferron-hexone-0.1 M NaClO $\mathrm{M}_{4}$ system. ${ }^{7}$ Two kinds of errors were tested on this system: a counting error $\sigma_{Q}(I)$ and a percentage error $\sigma_{Q}(\%)$. With 1 " $\sigma_{Q}(\mathrm{I})$ " the least squares method gave $\chi^{2} / k=412$, and of the four parameters $a_{0}$ came out negative, and $a_{0}$ and $a_{2}$ with standard deviations $>100 \% ; \chi^{2} / k=2791$ was obtained when only $a_{0}$ was omitted. Reasonable value of $\chi^{2} / k(0.95)$ was obtained from a weighing factor corresponding 
Fig. 2. The Th(IV)-cinnamic acid-chloroform-0.1 M $\mathrm{NaClO}_{4}$ system. The solid curve (-) is computed with the least squares parameters in Table $1 \mathrm{f}$. The dashed curve (....) was obtained with the parameters of the two-parameter method.

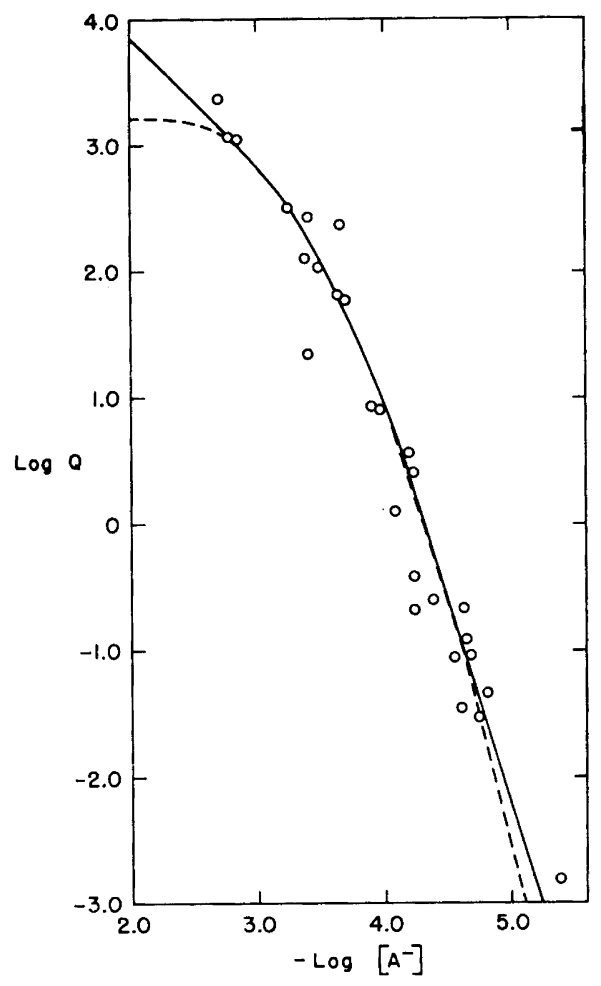

to 20 times " $\sigma_{Q}(\mathrm{I})$ ", indicating that the experiments involved much larger than the estimated counting errors.

When tested with the $\sigma_{Q}(\%)$ error, $a_{0}$ came out negative, and both $a_{0}$ and $a_{2}$ had standard deviations $>100 \%$. However, omitting $a_{0}$ only, $a_{2}$ came out with only a $75 \%$ standard deviation. Thus there is some support in the data for significance of the $a_{2}$ parameter. Reasonable $\chi^{2} / k=(0.91)$ was obtained for $\sigma_{Q}(\%)=0.4 Q$.

In Fig. 3 is plotted the experimental data and two curves, one obtained with the counting error and 2 parameters, and the other with the percentage error and 3 parameters. The shaded areas are the standard deviations in $Q$ due to the standard deviation in $a_{n}$. The two areas overlap each other. Since the curve with 3 parameters seems to fit the experimental data somewhat better, its parameter values are given in Table lg together with the equilibrium constants calculated from them. Within parenthesis, the $a_{n}$ values for the " $\sigma_{Q}(\mathrm{I})$ " computation are also given; it is seen that the two pairs of $a_{1}$ and $a_{3}$ values overlap each other.

h. The Sm(III)-cupferron-hexone-0.1 $\mathrm{M} \mathrm{NaClO}_{4}$ system. ${ }^{7}$ Both with a counting and a percentage error the least squares computations yielded a negative $a_{0}$ with a standard deviation $>100 \%$. A weight corresponding to 7 " $\sigma_{Q}(\mathrm{I})$ " produced $\chi^{2} / k=0.82$, and a $20 \% \sigma_{Q}(\%)$ error produced $\chi^{2} / k=1.29$,

Acta Chem. Scand. 14 (1960) No. 1 


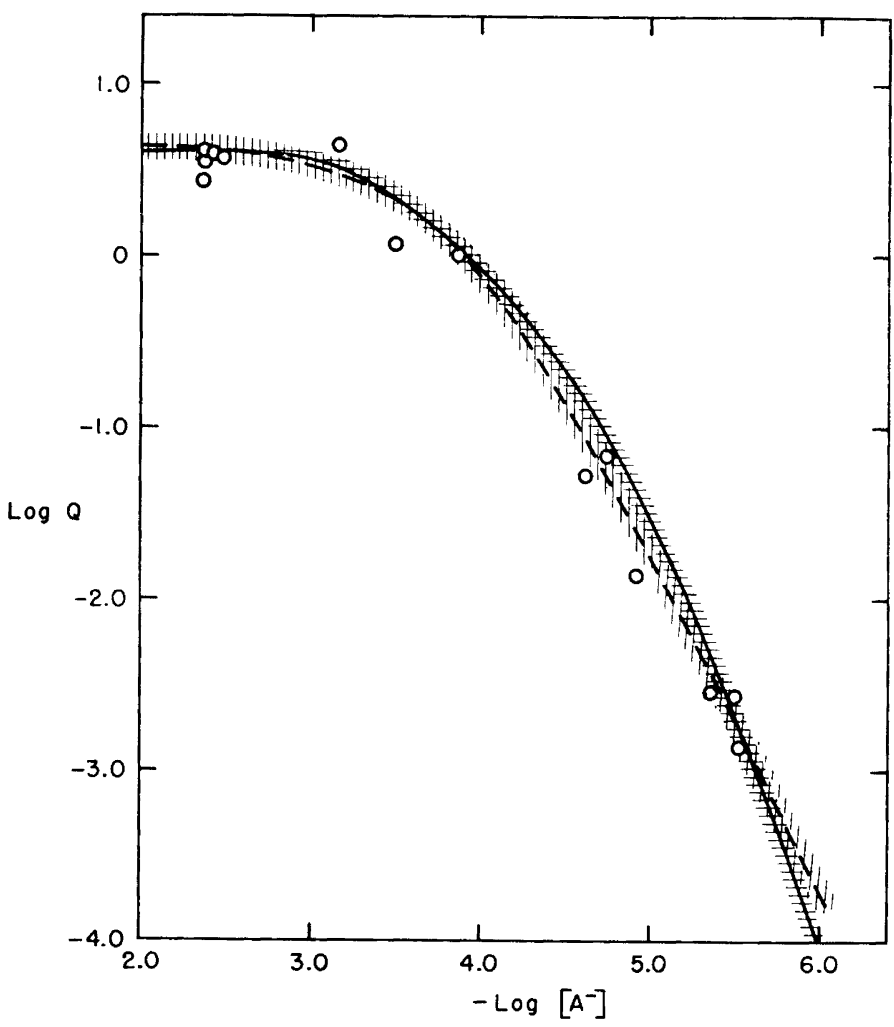

Fig. 3. The $\mathrm{La}(\mathrm{III})$-cupferron-hexone-0.1 M NaClO , system. The solid curve (- is obtained for a $\sigma_{Q}(I)$ error and 2 parameters, and the dashed curve (....) for a $\sigma_{Q}(\%)$ error and 3 parameters; see Table $1 \mathrm{~g}$. The shaded areas indicate the precision of the curves.

both cases with $a_{0}$ omitted. In Table th the parameters for the counting error are given with those for the percentage error within parenthesis; the differences between the two sets of parameters is negligible.

The least squares calculations indicate (in contradiction to the two-parameter method) that the experimental data do not allow a calculation of a $k_{1}$ value.

i. The Th(IV)-cupferron-chloroform-0.1 $\mathrm{M} \mathrm{NaClO}_{4}$ system. ${ }^{8}$ The least squares calculations were performed only with a counting error. A 10 " $\sigma_{Q}(\mathrm{I})$ " weight $*$ gave $\chi^{2} / k=1.66$ with the parameter $a_{1}$ negative, and $a_{1}$ and $a_{3}$ having standard deviations $>100 \%$. With $a_{1}$ and $a_{3}$ omitted, $\chi^{2} / k$ became 1.50; the corresponding $a_{n}$ values are given in Table li.

A comparison between the results of the two computational methods shows that more equilibrium constants than the data permit are calculated with the

* With 10 " $\sigma_{Q}(\mathrm{I})$ " weight, we mean a weight of $1 /\left(10 \text { " } \sigma Q(\mathrm{I})^{\prime \prime}\right)^{2}$. 
two-parameter method, and also that the precision of the $\lambda_{4}$ constant is overestimated.

j. The Th(IV)-dichlorooxine-hexol-0.1 $\mathrm{M} \mathrm{NaClO}_{4}$ system. ${ }^{9}$ Using a counting error in the least squares treatment, the parameters $a_{0}$ and $a_{2}$ came out with standard deviations $>100 \%$. For a 6 " $\sigma_{Q}(\mathrm{I})$ " weight, $\chi^{2} / k$ became 1.06 . The parameters obtained are given in Table $\mathrm{lj}$. The comments in paragraph $\mathrm{h}$ about the $k_{1}$ value are applicable also to this case.

k. The Th(IV)-methoxybenzoic acid-chloroform-0.1 $\mathrm{M} \mathrm{NaClO}$ system. $^{6}$ The least squares procedure using a 1 " $\sigma_{Q}(\mathrm{I})$ " weight gave $\chi^{2} / k=1.6 \times 10^{6}$ and all parameters with standard deviations in excess of $100 \%$. Therefore the weight of the points was decreased to correspond to a 40 " $\sigma_{Q}(\mathrm{I})$ " weight, where $\chi^{2} / k=4.5$ was obtained. With this weight all parameters except $a_{4}$ were negative or had standard deviations $>100 \%$. All combinations of 2,3 , and 4 parameters were tested; in no case was it possible to obtain a combination of only positive parameters with standard deviations $<100 \%$.

Since it was unreasonable to decrease the weight of the points further with this kind of error, the system was further investigated with a constant percentage error in $Q$. For $\sigma_{Q}(\%)=0.7 Q, \chi^{2} / k=1.4$ was obtained; of the five parameters only 2 were positive, but had standard deviations $>100 \%$. Again all possible combinations were run, and again no combination with only positive parameters with standard deviations $<100 \%$ could be obtained.

Obviously the experimental data are too poor to make possible any calculation of equilibrium constants with the least squares procedure and the present methods of weighing the data.

l. The Th(IV)-methoxybenzoic acid-hexone-0.1 $\mathrm{M} \mathrm{NaClO}_{4}$ system. ${ }^{6}$ The least squares procedure with a 30 " $\sigma_{Q}(\mathrm{I})$ " weight produced a $\chi^{2} / k=1.24$ with one parameter $\left(a_{1}\right)$ negative and one with a standard deviation close to $100 \%$ $\left(a_{3}\right)$. The system was therefore run, first with $a_{1}$ omitted, and then with all possible combinations of 2 and 3 omitted parameters. As a result, a number of possible combinations were obtained, all with a 30 " $\sigma_{Q}(\mathrm{I})$ " weight as shown in Table 3.

From this table it is seen that $\chi^{2} / k$ increases with decreasing number of parameters. Though the $\chi^{2} / k$ values in the last three cases is $>1.5$, they could rather easily be brought down to $<1.5$ by a slight decrease in weight; they are therefore worth being included in the Table.

Table 3. Possible parameter combinations for the Th(IV)-methoxybenzoic acid-hexone-0.I $\mathrm{M} \mathrm{NaClO}_{4}$ system when tested with a 30 "' $\sigma_{Q}(\mathrm{I})$ " weight (see paragraph 1).

\begin{tabular}{|c|c|c|c|c|c|c|}
\hline$\chi^{2} / k$ & $a_{0} \times 10^{13}$ & $a_{1} \times 10^{0}$ & $a_{2} \times 10^{6}$ & $a_{3} \times 10^{4}$ & $a_{4} \times 10^{2}$ & $\begin{array}{c}\text { Average stand. } \\
\text { dev. in } a_{n}\end{array}$ \\
\hline & & - & $1.25 \pm 0.34$ & $5.39 \pm 0.21$ & $3.9 \pm 1.8$ & $38 \%$ \\
1.31 & $1.04 \pm 0.39$ & - & - & $8.63 \pm 1.51$ & $2.7 \pm 1.8$ & $35 "$ \\
1.62 & $0.80 \pm 0.44$ & - & $1.94 \pm 0.25$ & $-17 \pm 0.4$ & $7.4 \pm 1.4$ & $29 "$ \\
1.65 & - & - & $1.61 \pm 0.37$ & $4.07 \pm 2.35$ & $4.5 \pm 2.1$ & $43 "$ \\
1.83 & - & - & $2.10 \pm 0.25$ & - & $7.2 \pm 1.5$ & $17 "$ \\
\hline
\end{tabular}


Considering both the $\chi^{2} / k$ values and the average standard deviations of all the $a_{n}$ values, some slight preference may be given to the combination yielding $\chi^{2} / k=1.35$. A plot of the curves obtained with the various parameter combinations will show that the curves for 3 and 4 parameters fit the experimental points about equally well. In view of the scatter of the data, the least squares method and the $\chi^{2}$ test cannot in this case distinguish between different functional models, and therefore no reliable equilibrium constants can be computed.

m. The Th(IV)-1-nitroso-2-naphtol-hexone-0.1 $\mathrm{M} \mathrm{NaClO}_{4}$ system. ${ }^{10}$ Using a counting error with the least squares method, $a_{0}$ was obtained with a standard deviation $>100 \%$. With the other 4 parameters and a 5 " $\sigma_{Q}(\mathrm{I})$ " wejght, $\chi^{2} / k=1.1$ was obtained. The corresponding parameter values are given in Table $1 \mathrm{~m}$.

In this case, like in some of the earlier ones, it is possible to omit an additional parameter and still obtain real values for the remaining parameters. The result, using the same weight is: $\chi^{2} / k=3.2, a_{1} \times 10^{26}=19.1 \pm 3.5$, $a_{3} \times 10^{9}=6 \pm 1$, and $a_{4} \times 10^{3}=6.4 \pm 3.7$. Since $\chi^{2} / k$ is considerably increased and the average standard deviation in the parameters is here $31 \%$ compared to $13 \%$ for the case with 4 parameters, it is concluded that, from a statistical point, the system is most correctly described with 4 parameters.

n. The Th(IV)-2-nitroso-1-naphtol-hexone-0.1 $\mathrm{M} \mathrm{NaClO}_{4}$ system. ${ }^{10}$ In all least squares tests, some parameters came out negative or with standard deviations $>100 \%$. The only possibly significant combination was obtained with the omission of $a_{0}$ and $a_{1}$, which gave $\chi^{2} / k=0.7$ for a 6 " $\sigma_{\text {(I) }}$ " weight. The parameters are given in Table $\mathrm{ln}$.

If also $a_{3}$ is omitted, one obtains for the same weights $\chi^{2} / k=2.9$ and the parameter values $a_{2} \times 10^{16}=2.12 \pm 0.21$ and $a_{4} \times 10^{3}=10.1 \pm 3.9$. Since $\chi^{2} / k$ as well as the average standard deviation in $a_{n}$ is greater with this parameter combination, the earlier results with 3 parameters are of greater statistical significance. However, a detailed analyses of the data shows that a higher statistical significance can be obtained using two parameters, $a_{2}$ and $a_{4}$, if the weight of one single point (at $-\log [\mathrm{A}]=6.41$ ) is considerably decreased. The least squares method does not allow a calculation of $k_{1}$ (cf. paragraph $\mathrm{h}$ ).

o. The $\mathrm{Sr}(\mathrm{II})$-oxine-chloroform-0.1 $\mathrm{M} \mathrm{NaClO}{ }_{4}$ system. ${ }^{11}$ In the original paper it is assumed that the organic phase only contains the complex $\mathrm{SrA}_{2} .2 \mathrm{HA}$. On this assumption the following polynomial is developed:

$$
\frac{[\mathrm{A}]^{2}}{Q}[\mathrm{HA}]^{2}{ }_{\text {org }}=\frac{1}{\beta_{2} \lambda_{2}{ }^{\prime \prime}}+\frac{\beta_{1}}{\beta_{2} \lambda_{2}{ }^{\prime \prime}}[\mathrm{A}]+\frac{\beta_{2}}{\beta_{2} \lambda_{2}{ }^{\prime \prime}}[\mathrm{A}]^{2}
$$

Using this expression in the least squares treatment, the parameters will become $a_{0}=1 / \beta_{2} \lambda_{2}{ }^{\prime \prime}, a_{1}=\beta_{1} / \beta_{2} \lambda_{2}{ }^{\prime \prime}$ and $a_{2}=1 / \lambda_{2}{ }^{\prime \prime}$, where $\lambda_{2}{ }^{\prime \prime}$ is the partition constant for the SrA $.2 \mathrm{HA}$ complex. For $\sigma_{Q}(\%)=0.3[\mathrm{HA}]^{2}$ org $/ Q, \chi^{2} / k$ is 0.89 and the following parameter values are found: $a_{0} \times 10^{3}=3.6 \pm 0.7$, $a_{1}=1.7 \pm 0.2$ and $a_{2} \times 10^{3}=1.0 \pm 6.0$. Since the standard deviation in $a_{2}$ is $>100 \%$, runs were made with $a_{2}$ omitted, yielding $\chi^{2} / k=0.86$ and the $a_{1}$ and $a_{0}$ values in Table lo; the $a_{0}$ and $a_{1}$ values are almost identical to those obtained with three parameters. 


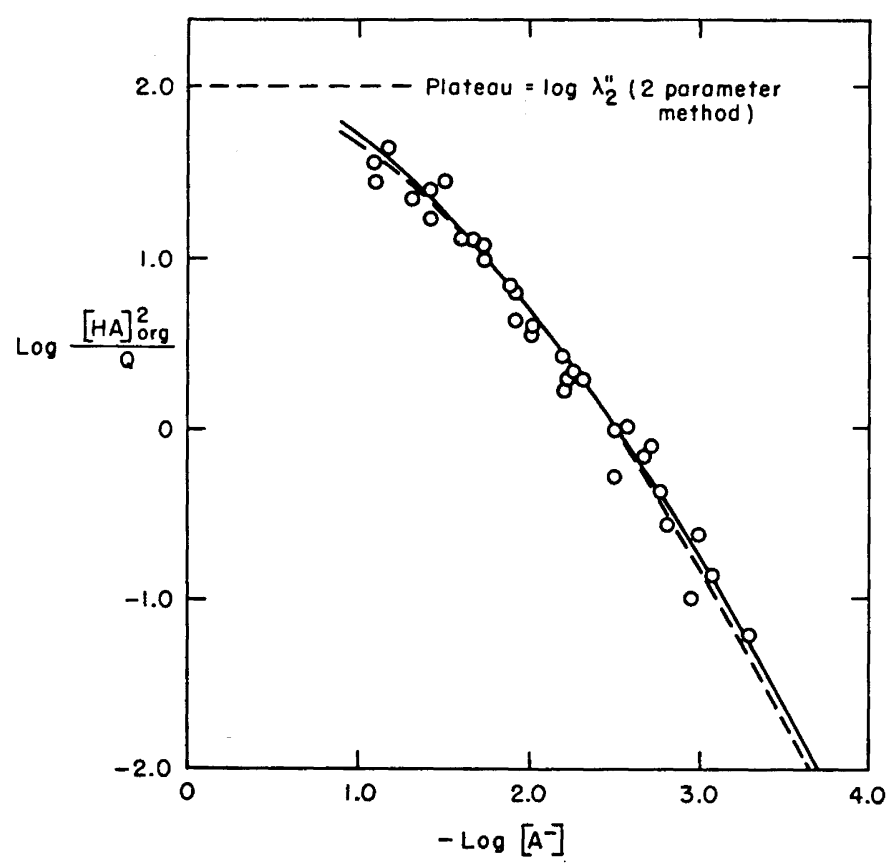

Fig. 4. The $\mathrm{Sr}$ (II)-oxine-chloroform-0.1 M NaClO 4 system. The solid curve (-) is obtained with the least squares parameters in Table $1 \mathrm{o}$, and the dashed curve $(. .$.$) with the parameters of the two-parameter method.$

From $a_{0}$ and $a_{1}$ only two equilibrium constants can be computed $k_{1}$ and $k_{2} \lambda_{2}{ }^{\prime \prime}$. With these 2 parameters, the solid curve in Fig. 4 is calculated. Since the curve goes smoothly through the experimental points, with about equally many points on both sides and without any tendency to level out into a sort of plateau, it is obvious that the data do not allow a separate calculation of the $\lambda_{2}{ }^{\prime \prime}$ value, as has been done with the two-parameter method.

p. The La (III )-oxine-chloroform-0.1 $\mathrm{M} \mathrm{NaClO}{ }_{4}$ system. ${ }^{7}$ All least squares computations using counting as well as percentage errors gave negative $a_{0}$, and $a_{0}$ and $a_{2}$ having standard errors $>100 \%$. Omitting these parameters, $\chi^{2} / k=1.08$ was obtained for a 20 " $\sigma_{Q}(\mathrm{I})$ " weight; the same $\chi^{2} / k$ value was obtained for a $70 \%$ error in $Q$. The parameters are given in Table lp. A comparison with the results of the two-parameter computation shows considerable discrepancies.

Fig. 5 gives the curves calculated with the parameters of the two computational methods. It is seen that the two-parameter curve has about equally many points on each side, while the least squares curve has about twice as many points below as above the curve. However, with badly scattered data the log scale is misleading, because in such a plot the average value will always be closer to the higher points $\left(e . g . y_{1}=5, y_{2}=15\right.$ and thus $\bar{y}=10$; therefore $\log y_{1}=0.7, \log y_{2}=1.18$, and $\log \bar{y}=1.0$ and not $\left.(0.7+1.18) / 2=0.94\right)$. 


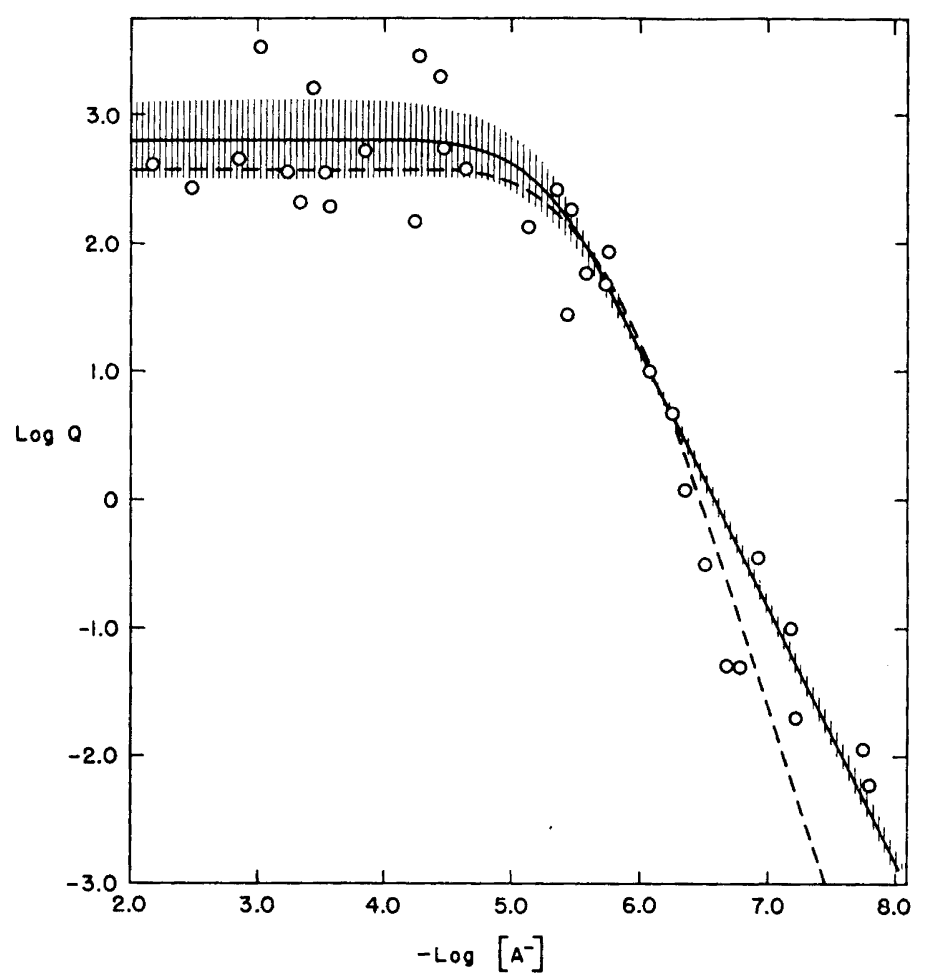

Fig. 5. The La(III)-oxine-chloroform-0.1 M NaClO $\mathrm{Nastem}_{4}$. The solid curve (is obtained with the least squares parameters of Table $1 \mathrm{p}$ (using 2 parameters), and the dashed curve (....) with the parameters of the two-parameter method (using 3 parameters). The shaded area indicates the precision of the least squares curve.

When the higher values are given a less weight than the lower ones the average curve will move closer to the lower points; this effect of weighing is not sufficiently pronounced in Fig. 5 to move the least squares curve down to the twoparameter curve (the author of the original paper has obviously weighted the higher points still less than the present author).

q. The Sm (III )-oxine-chloroform-0.1 $\mathrm{M} \mathrm{NaClO}_{4}$ system. ${ }^{7}$ Using percentage and counting errors for the least squares treatment, the parameters $a_{0}$ and $a_{2}$ came out negative and with standard deviations $>100 \%$. Omitting these parameters, the standard deviation in $a_{3}$ turned out to be $>100 \%$ when the counting error was used, but not with the percentage error. With $\sigma_{Q}(\%)=$ $0.5 Q$, a $\chi^{2} / k$ value of 1.35 was obtained. The corresponding $a_{n}$ values are given in Table lq. The discrepancies between the results of the two-parameter method and the least squares method are again quite large.

$r$. The Th(IV)-oxine-chloroform-0.1 $\mathrm{M} \mathrm{NaClO}$ system. ${ }^{8}$ Because of the big range of free ligand concentration $\left(10^{-1} \gtrsim[\mathrm{A}] \gtrsim 10^{-11}\right)$, the GEORGE computer could not handle the whole problem for $N \cong 6$. It was therefore decided 
to split it into two parts and run these two separately with overlapping [A] values (the same principle of splitting was used in the original paper with the two-parameter method). This splitting can be justified, because the $Q$ values are almost independent of $[\mathrm{A}]$ in the range $10^{-4}>[\mathrm{A}]>10^{-8}$, which means that in the presence of negatively charged complexes one can neglect the positively charged ones, and vice versa. Considering the experimental data in the original paper, the problem was divided in the two following parts:

Split I: $z=\sum_{0}^{4} a_{n} x^{n}$, run on the IBM 704 for $x<10^{-3}$.
Split II: $z=\sum_{0}^{2} a_{n+4} x^{n}$, run on the GEORGE for $x>10^{-9}$.

Using a percentage as well as a counting error in the Split I case, $a_{1}$ came out negative. Omitting this parameter, $a_{0}$ was obtained with a standard deviation $>100 \%$ (this $a_{0}$ is given within parenthesis in Table $1 r$; it is used for computing an approximate $k_{1} k_{2}$ value). Omitting both $a_{0}$ and $a_{1}$, the parameters in Table $1 r$ were obtained; the $70 \%$ error in $Q$ yielded a $\chi^{2} / k=0.83$, and the 17 " $\sigma_{Q}(\mathrm{I})$ " weight $\chi^{2} / k=0.98$.

Only a $70 \%$ error was tested in the Split II case, which first yielded a negative $a_{6}$ value with standard deviation $>100 \%$. With this parameter omitted, the $a_{4}$ and $a_{5}$ values in Table Ir were obtained with a $\chi^{2} / k=0.73$. The good agreement between the $\chi^{2} / k$ and $a_{4}$ values of Split I $\left(\sigma_{Q}(\%)\right)$ and Split II $\left(\sigma_{Q}(\%)\right)$ indicates consistency between the two split cases.

The equilibrium constants in Table Ir are computed from the $\sigma_{Q}(\%)$ data, $\lambda_{4}$ being taken from $a_{4}$ of Split I $\left(\sigma_{Q}(\%)\right)$. A comparison between the results of the two computational methods indicates that more parameters (and more equilibrium constants) are given by the two-parameter method than the experimental data justify. Particularly dubious is the calculation by that method of a $k_{1}$ value, because in the concentration range where the first complex, $\mathrm{MA}^{3+}$, is supposed to exist, the scatter of the experimental data is tremendous $\left(e . g\right.$. for $-\log \left[\mathrm{A}^{-}\right]=10.53 \pm 0.01$ the spread in $\log Q$ is $1.75, i . e$. over a factor of 50 in $Q$ ).

Because of the generally very great scatter of experimental data in this system, the comments in $\S \mathrm{p}$ about the distortion of the log scale is particularly applicable to this case.

s. The $T h(I V)-N$-phenylbenzohydroxamic acid-chloroform-0.1 $\mathrm{M} \mathrm{NaClO}_{4}$ system. ${ }^{12}$ With a counting error, the least squares calculations yielded a negative $a_{1}$, and $a_{1}$ and $a_{3}$ with standard deviations $>100 \%$. Omitting these parameters, a $\chi^{2} / k=0.91$ was obtained with a 20 " $\sigma_{Q}(\mathrm{I})$ " weight; the resulting parameters are given in Table 1s.

The average standard errors given for $p_{1}$ and $p_{2}$ is somewhat above $12 \%$, while for $a_{0}$ and $a_{2}$ it is about $40 \%$. Similarily, for $\lambda_{4}$ the two-parameter method gives a standard error of about $7 \%$, while the least squares method gives about 
$33 \%$. The high precision given for the two-parameter method seems therefore overestimated.

t. The Th(IV)-salicylic acid-hexone-0.1 M NaClO $\mathrm{M}_{4}$ stem. ${ }^{\circ}$ Whith the functional form given for this system in the original paper, the same equations as before can be used in the least squares treatment, provided $Q$ is replaced by $Q /\left[\mathrm{H}_{2} \mathrm{~A}\right]_{\mathrm{aq}}$ and the partition constant $\lambda_{4}$ is replaced by

$$
\lambda_{4}^{\prime}=\frac{\left[\mathrm{Th}(\mathrm{HA})_{4} \mathrm{H}_{2} \mathrm{~A}\right]_{\mathrm{org}}}{\left[\mathrm{Th}(\mathrm{HA})_{4}\right]_{\mathrm{aq}}\left[\mathrm{H}_{2} \mathrm{~A}\right]_{\mathrm{aq}}}
$$

where $\mathrm{H}_{2} \mathrm{~A}$ stands for undissociated salicylic acid. With the least squares treatment using a percentage error in $Q$, the parameters $a_{1}$ and $a_{3}$ came out with standard deviations $>100 \%$. Omitting them and using $\sigma_{Q}(\%)=0.3 . Q$, a $\chi^{2} / k$ value of 0.94 and the parameter values in Table lt were obtained. The table indicates that more equilibrium constants have been computed from the two-parameter results than the experimental data permit.

$u$. The Th(IV)-tropolone-chloroform-0.1 $\mathrm{M} \mathrm{NaClO}$ system. ${ }^{13}$ With this system the same mathematical difficulties were encountered as with the $\mathrm{Th}(\mathrm{IV})$-oxine system in $\S \mathbf{r}$, and the same splitting procedure was used to get around these difficulties; Split I was used for [A] $<10^{-2}$, and Split II for [A] $>10^{-7}$.

Using a percentage and a counting error, $a_{0}$ and $a_{3}$ came out negative, and $a_{3}$ with a standard deviation $>100 \%$. Omitting $a_{0}$ and $a_{3}$, the parameters $a_{1}, a_{2}$ and $a_{4}$ given in Table lu were obtained; for a $70 \%$ error in $Q, \chi^{2} / k=$ 0.51 was obtained, while a counting error corresponding to 15 " $\sigma_{Q}(\mathrm{I})$ " gave $\chi^{2} / k=0.73$. It is seen in the table that the equilibrium constants $k_{2}$ and $k_{3} k_{4}$ computed from the two Split I data agree well (the values within parenthesis are computed from the Split I $\left(\sigma_{Q}(\%)\right)$ data), while the precision of the $k_{3} k_{4}$ and $\lambda_{4}$ values obtained from the Split I $\left(\sigma_{Q}(\mathrm{I})\right)$ data is less good.

The Split II case was only run with a percentage error in $Q$, which gave an $a_{5}$ value with a standard deviation $>100 \%$. Omitting $a_{5}$ and using a $\sigma_{Q}(\%)=$ $0.7 Q$ error, a $\chi^{2} / k$ value of 0.56 and the parameters $a_{4}$ and $a_{6}$ in Table lu were obtained; $\log k_{5} k_{6}$ in the table is computed from these $a_{4}$ and $a_{6}$ values. It is seen that the $70 \%$ error in $Q$ used in both Split I $\left(\sigma_{Q}(\%)\right)$ and Split II $\left(\sigma_{Q}(\%)\right)$ give approximately identical $a_{n} \pm \sigma_{a_{n}}$ and $\chi^{2} / k$, demonstrating good consistency between the two treatments.

With the equilibrium constants of the least squares procedure, a curve $Q([\mathrm{~A}])$ can be computed which goes smoothly through the experimental points. Thus, with the present spread in the experimental data, the Th(IV)-tropolone system can be completely explained by only 4 equilibrium constants.

\section{DISCUSSION}

With good data, the ligand number and limiting value methods should give the same equilibrium constants as the least squares method; cf. Ref. ${ }^{15}$ This is also true for the two-parameter method as long as only two complexes are present in the system studied. 
Though only the least squares method yields true standard deviations of the constants, rather good estimates of precision can be made with the limiting value method; these two methods are the only ones here discussed where the experimental data are used directly for computing the parameters. On the other hand, precision estimates are very hard to make with the ligand number method, because the calculation of the complexity constants are based on points $\bar{n}$ ([A]) taken from a smoothed curve through the experimental points (cf. discussion in connection with eqn. 5). This is rather pointedly illustrated by comparing the dissertations of $\mathrm{J}$. Bjerrum ${ }^{16}$ (ligand number method and few estimates of precision) and I. Leden ${ }^{18}$ (limiting value method and almost all constants with precision estimates). Good estimates of precision are also hard to make with the two-parameter method.

With very scattered data, as has been the case in the solvent extraction work here investigated, different results may be anticipated with different computational methods.

In the ligand number method, the graphical differentiation of the smooth curve through the experimental points may introduce errors which in the later computations cannot be detected; this method may therefore sometimes lead to the calculation of more constants than the original data would permit (see Tables $\mathrm{lb}$ and $\mathrm{d}$, and Ref. ${ }^{1}$ ).

The limiting value method is sensitive to large spread of the experimental data. With poor data it is extremely hazardous to extrapolate curves through the measured or calculated points to zero. Further, the errors obtained in one constant propagates to the successive constants. If the computations are not carried out carefully, such a propagated error may in a later step turn up as a "constant". Though the limiting value method makes more direct use of the experimental data than any of the other graphical methods, its use should be limited to rather good data.

With very poor data, the visual judgement of the two-parameter method may be the only way to get information about the system. In such a case one should realize that the method is entirely qualitative (see paragraphs $\mathrm{k}$ and 1 in the experimental part).

The two-parameter method is based on the assumption that the experimental data fall on a curve with two asymptotes, one with slope zero (when the aqueous phase contains almost only the uncharged complex $\mathrm{MA}_{\nu}$ ) and one with slope $v$ (when the aqueous phase contains almost only uncomplexed metal ions $\left.\mathrm{M}^{\nu+}\right) *$ When this method is carelessly applied to data which do not closely approach these asymptotes, dubious results may be obtained. This is demonstrated in a great number of tables above, where from the two-parameter results partition constants $(q$ 's $)$ and $k_{1}$ or $k_{1} k_{2} \ldots . . k_{N}$ have been calculated, while the least squares method shows that there is no support in the experimental data for significant values of these constants. ** Thus improper application of the two-parameter method may produce more parameters than the experimental data allow (Tables le, f, o, p, q, r and u); on the other hand,

\footnotetext{
* The principles of the two-parameter method can be used with the assumption that the first measurable complex is $\mathrm{MA}_{r}$, giving an asymptote of slope $\nu-r$; however, this has never been done.

** By definition (see equations 2 and 8) $k_{1} k_{1} \ldots \ldots \ldots k_{N}=10 N p_{1}$.
}

Acta Chem. Scand. 14, (1960) No. I 
with very good data the method does not always permit the evaluation of all parameters involved (Tables $1 \mathrm{c}$ and $\mathrm{m}$ ).

A draw-back of the two-parameter method is also the difficulty in relating the parameters to any specific equilibrium constants (cf. Tables li, s and $t$, where the two different computational methods have produced the same number of parameters, and $k_{1}$ and $\lambda_{\nu}$ both exist). Though there is some experimental support for the existence of a constant spreading factor, cf.e.g. Ref. 16,18, certainly not all systems are of the regular type indicated by eqn. 9. The calculation of step-wise equilibrium constants from the parameters of the twoparameter method must therefore be based on additional information about the system than the experimental data alone provide. In fact, such a calculation is in contradiction to the original purpose of the two-parameter method ${ }^{14}$.

The simultaneous consideration of all experimental data in the least squares method leads to the computation of the maximum number of significant equilibrium constants and their precision; in this respect this method is superior to any of the graphical methods.

However, graphical plots must be made before the least squares calculations are begun, in order to see if any curve at all can be fitted to the experimental points; it is also advisable to check the results of the computations with the input data, so that errors in making up the programs, etc. are disclosed, cf. Ref. ${ }^{15}$

When parameters are omitted in the least squares calculations (because they are negative or their standard deviations exceed the parameter value) this does not necessarily mean that the corresponding complexes do not exist, but only that the equilibrium constants cannot be calculated because of uncertanties in the experimental data (cf. paragraph d).

Since the laborius manual computations of the least squares method are now overcome by the use of high speed digital computers, it is hoped that this method will be more used in the future.

The present investigation also indicates the need for more refined technique in solvent extraction work.

Acknowledgement. The author wishes to thank Drs. J. C. Hindman and J. C. Sullivan of the Chemistry Division, and Dr. W. Miller, Mr. W. Greenhow and Mrs. M. Hanson of the Applied Mathematics Division, for their extensive cooperation in this investigation. $\mathrm{He}$ is also most grateful to Dr. W. M. Manning, Head of the Chemistry Division, for his interest in this work and for the research appointment which made it possible.

\section{REFERENCES}

1. Rydberg, J. and Sullivan, J. C. Acta Chem. Scand. 13 (1959) 2057.

2. Rydberg, J. Acta Chem. Scand. 4 (1950) 1503.

3. Rydberg, J. Arkiv Kemi 5 (1953) 413.

4. Rydberg, J. and Rydberg, B. Arkiv Kemi 9 (1956) 81.

5. Rydberg, J. Arkiv Kemi 9 (1956) 109.

6. Hök-Bernström, B. Acta Chem. Scand. 10 (1956) 174.

7. Dyrssen, D. Svensk Kem. Tidskr. 66 (1954) 234.

8. Dyrssen, D. Svensk Kem. Tidskr. 65 (1953) 43.

9. Dyrssen, D., Dyrssen, M. and Johansson, E. Acta Chem. Scand. 10 (1956) 341.

10. Dyrssen, D., Dyrssen, M. and Johansson, E. Acta Chem. Scand. 10 (1956) 106.

Acta Chem. Scand. 14 (1960) No. 1 
11. Dyrssen, D. Svensk Kem. Tidskr. 67 (1955) 311.

12. Dyrssen, D. Acta Chem. Scand. 10 (1956) 353.

13. Dyrssen, D. Acta Chem. Scand. 9 (1955) 1567.

14. Dyrssen, D. and Sillén, L. G. Acta Chem. Scand. 7 (1953) 663.

15. Sullivan, J. C., Rydberg, J. and Miller, W. Acta Chem. Scand. 13 (1959) 2023

16. Bjerrum, J. Metal Ammine Formation in Aqueous Solutions, Diss. Copenhagen 1941.

17. Sullivan, J. C. and Hindman, J. C. J. Am. Chem. Soc. 74 (1952) 6091.

18. Leden, I. Potentiometrisk undersökning av några kadmiumsalters komplexitet, Diss. Lund 1943.

19. Rydberg, J. and Sullivan, J. C. Acta Chem. Scand. 13 (1959) 186.

20. Deming, W. E. Statistical Adjustment of Data. J. Wiley \& Sons, New York 1948.

Received August 6, 1959. 